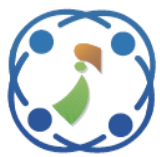

\title{
An Improved Behavioral Biometric System based on Gait and ECG signals
}

\author{
Bouchiba Guelta ${ }^{1 *}$ \\ Redouane Tlemsani ${ }^{2}$ \\ Samira Chouraqui ${ }^{1}$ \\ Mohamed Benouis ${ }^{3}$ \\ ${ }^{l}$ Département d'informatique, Université des Sciences et de la technologie Mohamed-Boudiaf, Oran, Algeria \\ ${ }^{2}$ Tronc Commun Department, National Institute of Telecommunications and ICTs of Oran, Oran, Algeria \\ ${ }^{3}$ Computer Science Department, Mohamed Boudiaf University of Msila, Msila, Algeria \\ * Corresponding author's Email: bouchiba.guelta@univ-usto.dz
}

\begin{abstract}
This paper presents multi-modal biometric authentication approach using gait and electrocardiogram (ECG) signals, which can diminish the drawback of unimodal biometric approach as well as to improve authentication system performance. In acquisition phase, data sets are collected from three different databases, ECG-ID, MIT-BIH Arrhythmia database and UCI Machine Learning Repository (Gait). In Feature extraction phase of both signals (ECG and Gait) is performed by using 1D-local binary pattern. Features are obtained by merging two modalities as one feature. In classification approach, three classifiers are developed to classify subjects. K-nearest neighbour (KNN), relying on Euclidean distance, PNN (Probabilistic Neural Network), RBF (Radial Basis Function) and Support Vector Machine (SVM), relying on One-against-all (OAA). The proposed multimodal system has been tested over 18 subjects, and its identification accuracy was about $100 \%$. Our result demonstrate that our approach outperforms rather than unimodal biometric system in terms of Correct Recognition Rate, Equal Error Rate, False Acceptance Rate and False Reject Rate.
\end{abstract}

Keywords: Multibiometric system, ECG, GAIT, 1D- LBP, PNN, KNN, SVM, RBF.

\section{Introduction}

All Multibiometric system using different modalities is a very challenging task because the unimodal system shows many lacks (i.e., such as noisy data, intra-class variability, inter-class similarities etc.) in different application task. In contrast, human behaviour gained much attention as a means of identifying a subject from the dynamics of some of its signals. This produced challenging questions, opening a broad area of signals to explore and new methodologies to propose in answering a difficult problem: How to extract, combine and classify characteristics from dynamic sources to distinguish many classes.

Designing an efficient biometric system robust to realistic context conditions is a challenging computer vision problem with many applications, including data acquisition and feature extraction or machine learning [1]. Over the last decade, biometric system based on fingerprint, iris and face were have been used successfully. However, many people felt unsuitable (unfriendly) under the constraints that required to perform the identification task of human being [2].

On other hands, no biometrics is reliable 100\%, which make any unimodal biometric system still binding. Some of the challenges commonly encountered by these systems such as the problem of environment in which the captured data may be affected by illumination, pose, noised data and other effects closely related to the types of biometric technology [3]. Due to improperly maintained sensors, spoofing attacks: the unimodal biometric makes its mimic easier from an imposter. In addition, Non-universality is due to the absence of acquired traits or its poor quality because of accidents for example.

According to the limitations listed above imposed by conventional biometric systems, performance can be enhanced by using dynamic action instead of physical traits. The growing number of users of smart 
device is resulting in an increasing amount of private information being stored inside each such devices. Numerous problems in security and privacy are constantly being raised. To resolve these issues, researchers have implemented many methods including continuous authentication approaches based on user behaviour [4].

Recently, biometric technologies have moved towards more real-time, remote, distance-based, noncooperative and non-invasive surveillance. ECG and gait recognition can form the basis of unobtrusive technologies for the detection of individuals who represent a security threat or behave suspiciously. A behavioural biometric solution would be advantageous to screen individuals in high-security civilian or military facilities and healthcare monitoring of human being [5].

In modern medical applications, the desire for efficient biomedical systems along with the problem of increasing health costs has moti-vated the development of biopotential electrodes [1,3], smart bio-sensing devices [4], smart textile materials [5] [6], microelectronics circuits [7] and smart wireless communications techniques [8].

One of the earliest works that examined ECG electrode for biometric systems dates back to 1977 [6]. However, a clean ECG signal requires the use of clean room facilities and bulky equipment thereby making the manufacturing process relatively expensive and time-consuming [7]. In general, healthy ECG heartbeat has six characteristic or fiducial points, namely $\mathrm{P}, \mathrm{Q}, \mathrm{R}, \mathrm{S}, \mathrm{T}$, and $\mathrm{U}$. These points are illustrated in Fig. 4. Based on these fiducial points, two mainstreams of ECG analyses have been proposed in the literature: fiducial and non-fiducial points-based approaches. ECG heartbeats are isolated from ECG signals then aligned together to have a persistent feature extraction. Most of the techniques, whether they are fiducial or non-fiducial points-based approaches, perform heartbeat isolation and alignment. Some exceptions exist such as the works in [8-11]. In fiducial points-based approaches, features are extracted from fiducial points. Features can be distances between points, angles, areas, amplitudes, etc. One of the most pertinent works to this paper in the fiducial points-based approach is in [12] because it deploys sequential sampling to make verification biometric decision.

Now days, Gait biometric for human walk manner recognition has been gaining a more attention of many research laboratory because of its strengths especially in security domain, medicine and psychology, disorder prevention [13-15].

In the literature, the proposed works in the gait biometric are generally categorized on three major approach based on the sensor, which is used to record the gait: machine vision-based, floor sensor-based and wearable-based [16]. C. BenAbdelkader, R. Cutler, H. Nanda, et L. Davis [17] have firstly studied the gait for human identification where he proposed an eigen gait method using image self-similarity plots.

After that, several works have been developed where were using both the model free based and model based to extract the information's that describe the manner of human walk [18-20].

I. Rida, N. Almaadeed, and S. Almaadeed [21] give a comprehensive discussion about the state of the art in vision-based gait recognition up to 2018.

On other hand, research on accelerometer-based gait recognition started in 2005 by H. J. Ailisto, M. Lindholm, J. Mantyjarvi, E. Vildjiounaite and S.-M. Makela [22] and was further investigated by $D$. Gafurov [23]. In the initial stages, dedicated accelerometers were used and worn to different body parts like the feet, hip, arm or ankle. Only recently researchers started to use smart phones as" sensors" [24]. I. Dehache and L. Souici-Meslati in [25] proposed an accelerometer-based biometric gait recognition by feeding the accelerometer data on immunological classifiers to identify the subjects.

For instance, several multimodal biometric systems based on conventional traits such as; face fingerprint and irises etc. have been developed during past decades in [26,27,28,29]. so far, there are only a few works about a multimodal biometric system that includes behavior with physical traits (M. Derawi and I. Voitenko 2014, N. Belgacem, R. Fournier, A. NaitAli, and F. Bereksi-Reguig 2015, M. Regouid, M. Touahria, M. Benouis and N. Costen 2019; M. Hammad and K. Wang 2019) [30-33].

The literature on bimodal or multimodal biometrics is vast; however, there is very little research on the combination of ECG and Gait biometrics. This is possibly due to the fact that ECG and Gait data is mostly low massive. Consequently, the most of existing multimodal systems combine either the ECG or Gait with the conventional biometric such as Face, iris, fingerprint and so on.

As well as environment and security contexts, research has demonstrated that such physical trait (i.e; face iris, image gait) is sensitive to aspects such as diseases, age, large variations in illumination and facial expressions, clothing and others challenges. Therefore, it is reasonable to believe that combining the behavioral biometrics would improve the recognition accuracy.

Gait recognition has emerged in several studies [17-19] due to its strength, robustness and efficiency in surveillance identification. ECG biometrics has acquired a large interest because of its benefits such 
as a stable structure, robust information and rich features. On other hand, Also, both ECG and gait are hidden biometric; both can be extracted through the same sensor, precluding the need for separate or specialized equipment [34].

To build up the aforementioned system, fusion scheme is an important part of a multimodal biometric system. A fusion is a manner of combining the information from multiple domain experts. So, the aim of the fusion is to estimate the best set of experts for a given problem domain [35]. There are various traditional fusion rules for multimodal biometric systems: rank level fusion, decision level fusion, feature level fusion and match score level fusion. The rank level fusion computes the final rank of a user based on the ranks obtained from the individual classifiers, and various techniques such as logistic regression are utilized to make the final decision based on the rank of each identity [36]. The decision level fusion takes the final decision of acceptance and rejection in various ways, such as majority voting scheme, weighting the majority voting, Bayesian fusion, etc. In feature fusion, fusing is done before matching, and features of all the modalities are combined and then the final decision is made after matching. In match score fusion, multiple classifiers are used to set the match scores for each modality that are fused to form a final scalar score. In this work, we have followed the match score level fusion to set up both modalities and its has a proven performance record. The authentication via the modern smartwatch contains multiple sensors offers a user-friendly alternative to common authentication methods on smartphones [37]. It has the great advantage that the authentication can be performed without user interaction. When the user is walking, his walk-pattern can be extracted from the accelerations measured using the integrated sensors of the smartphone and his activity of heart can be extracted from the ECG recorded using the smartwatch [38]. Those patterns can be easily fused and used for authentication.

Generally, each biometric system contains an important stage named; feature extraction. Indeed, several feature extractions of methods are used both for ECG and gait signal including temporal series, Time-Frequency domain, and statistical analysis. Local binary pattern [39] also known as features extraction, represent a well-known approach in the machine vision field. This technique is interesting because it exhibits many properties that can be incorporated into many application systems: biometric, texture analysis, face detection $[40,41]$. The advantage of using LBP feature is to extract the prominent information from the heartbeat ECG and magnitude acceleration of a Gait signal. This work also demonstrates the high suitability of using the LBP features for both modalities ECG and Gait. Moreover, the proposed approach investigated the advantages of both modality's, ECG and Gait as a new fused data and therefore to give us more confidence and high accuracy instead of the unimodal biometric analysis of 1D signals is an emerging and potentially very important application area for LBP. For example, ECG based biometric systems such as identification task require robust and inexpensive signal processing.

\section{The Proposed approach}

Signals of Human behavioural from their activity were collected using wither heart activity or manner of walking investigated in the context of the present paper. In the following, we describe the acquisition process of ECG and Gait signal, the extraction of the corresponding features and finally the definition of the new biometric proposal by fusing the extracted ECG and accelerometer-based gait signals. In Fig. 1 the steps involved in the system are summarized Printing Area.

The printing area is $125 \mathrm{~mm} \times 197 \mathrm{~mm}$. The text should be justified to occupy the full line width, so that the right margin is not ragged, with words hyphenated as appropriate. Please fill pages so that the length of the text is no less than $180 \mathrm{~mm}$.

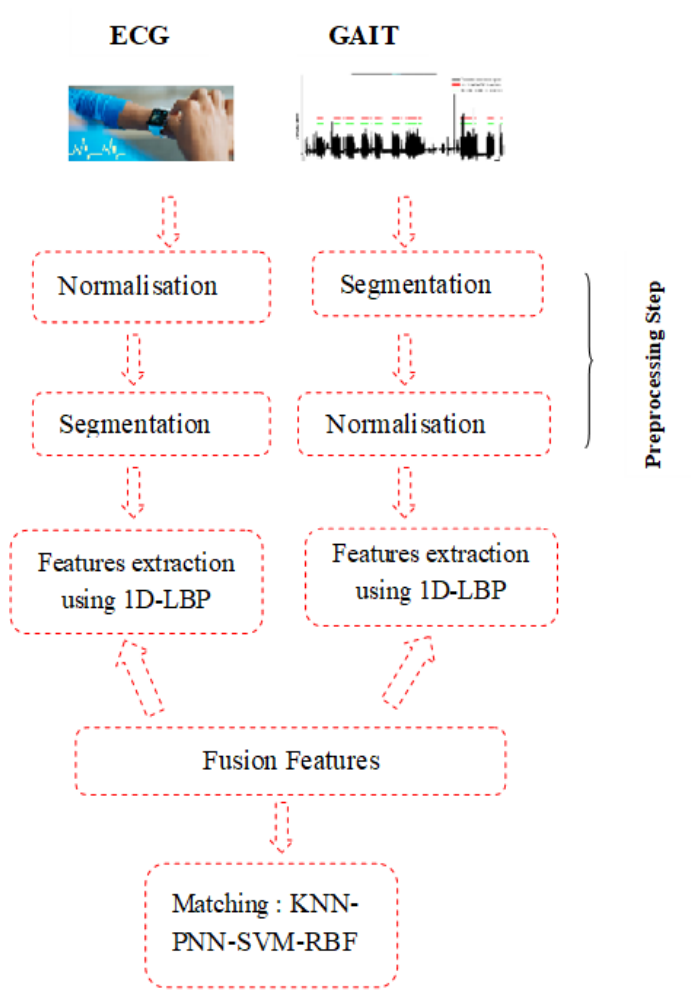

Figure.1 The proposed recognition system 
Even though many methods of feature extraction from ECG and Gait signals have been well done to discriminate among different signals, one of the major issues attached with it is the computational performance of the feature extraction techniques. The techniques based on local transformation are computationally simple and extract features with higher discriminating power. The success of the LBP in 2D and 3D applications motivated us to utilize it in the gait and ECG identification problem. In this regard, few researchers considered using LBP in ECG recognition either as a stand-alone signal representation technique or combined with other methods. In [42], the LBP descriptor is used to extract ECG fiducial features. The performance is tested using ECG signal for 90 subjects from ECG-ID dataset. For the gait recognition, [43]. El-Alfy studied the gait-based gender problem by using a modified method of fuzzy local binary patterns for feature extraction and SVM for the prediction the gender of walking person. Omar investigated a novel descriptor for gait recognition known as Kernel-based Fuzzy Local Binary Pattern (KFLBP). Ertuğrul [44] proposed a new local pattern transformation technique namely one shifted local binary pattern. This latter was proposed to identify the Parkinson's disease from gait signal of human being. We explain in detail the steps of our system; preprocessing, feature extraction step, fusion features and classification steps.

\subsection{Preprocessing stage}

This stage includes two sub-steps for each biometric modality which consist of pre-processing and segmentation for both ECG and Gait signal.

For ECG signal, Pre-processing stage aims to reducing the noise from the ECG signal and removes various artefacts and improves the signal equality by applying SG-FIR (Savitzky-Golay Finite impulse response) technique [45]. Fig. 3 shows about 10 second from the original ECG signal and its preprocessed one in Fig. 2. In this stage, a segmentation of ECG signal was done by located the PQRST points. In our work, we have adopted PanTompkins algorithm [46] to isolate the fiducial point $(\mathrm{P}, \mathrm{Q}, \mathrm{R})$ for each beat segment. Each detected Rpeak determines the center of the QRS complex. Then, to isolate the heartbeat, we take 94 samples before the R-peak and 150 samples after the R-peak which means that each ECG heartbeat have 245 samples and $490 \mathrm{~ms}$ segment duration. Fig. 4 shows segmented heartbeats for the same and different subjects aligned with the $\mathrm{R}$ peak.
For the Gait signal, the segmentation can be conducted in two different ways. Either the segments are based on gait cycles or are determined by using fixed-length time windows. In our work, we have followed the second step where the data can be divided into segments of a fixed time length without considering the course of the signal. Segment lengths between 2 and $7.5 \mathrm{~s}$ are used in this thesis, the segments overlap by $50 \%$. Examples of these signals collected from 10 different individuals, and used for biometric classification, are presented in: Fig.2 (the gait signals); Fig. 3 (the ECG signal); and Fig. 4 (the one heartbeat signal).

\subsection{Features extraction}

Following pre-processing, feature extraction is carried out to extract the unique ECG and gait features the can distinctively describe an individual. In the current study, Local Binary Pattern (LBP) is

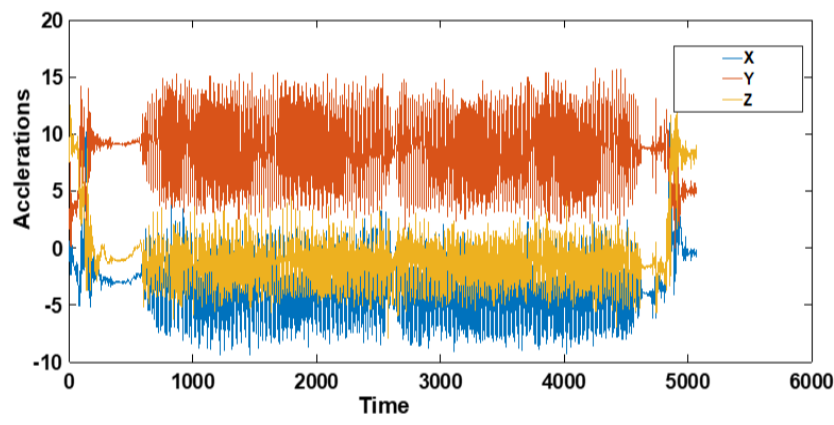

Figure.2 Raw accelerometer data of Gait signal

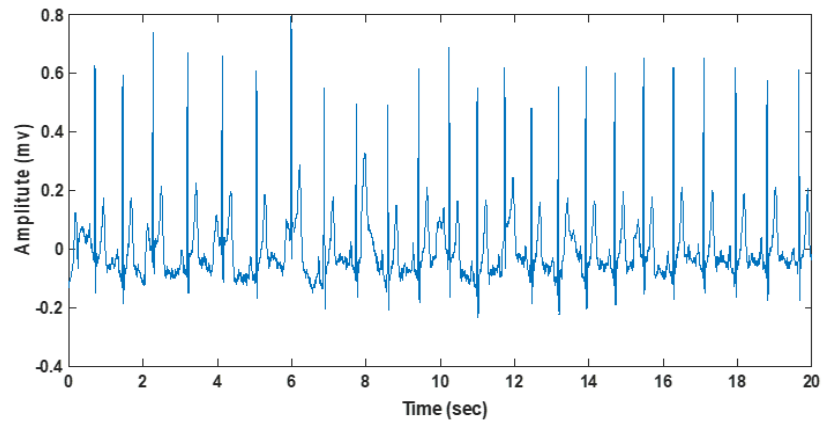

Figure.3 Original ECG signal

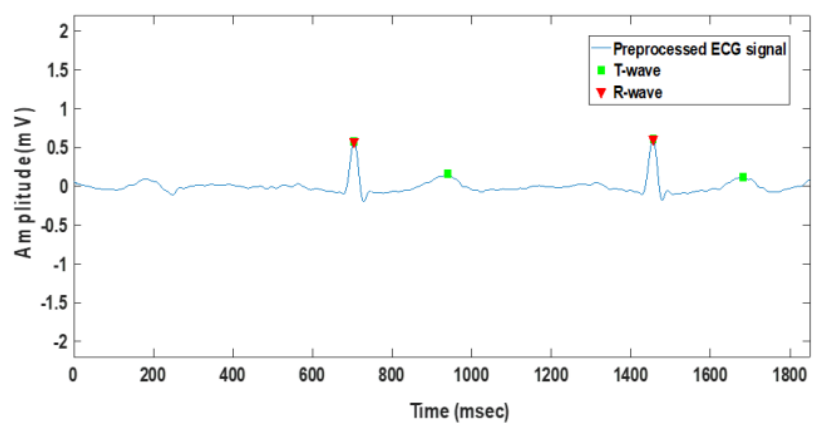

Figure.4 A segment of sample ECG signal 
used as the feature extraction method. 1D-LBP was successfully adopted over time series data in various studies [41]. 1D-LBP seeks to the important features of a data segment by taking the difference between center point with the right and left values of the center. All neighbor values are compared with the center value; if it is greater or equal it assigns as one, otherwise, it becomes zero. Next, all neighbor values are combined to form a binary representation of the original signal with respect to the center point.

Then, the binary value is converted to the decimal value as in Eq. (2). At this stage, each point on the original signal corresponds to its decimal value (see Fig. 6). By taking histogram, 1D LBP feature vector is formed. Eq. 2 summarizes the whole procedure.

$$
\begin{aligned}
S^{1 D-L B P}= & \sum_{i=0}^{m-1} \operatorname{Sig}\left(P_{i}-S_{c}\right) 2^{i} \\
& \text { Where } \operatorname{Sig}(x)=\left\{\begin{array}{c}
1, \text { if } x>0 \\
0, \text { otherwise }
\end{array}\right.
\end{aligned}
$$

In Eq. (1), $P_{i}$ gives values of neighboring samples and $S_{c}$ represents the center point.

For Gait signal, features are extracted from the $\mathrm{x}-, \mathrm{y}-$ and $\mathrm{z}$ - direction as well as from the magnitude vector.

$$
V=\sqrt{x^{2}+y^{2}+z^{2}}
$$

Once the magnitude vector was computed, we have performed 1D- LBP method to extract from them the significant features.

For each fixed-length time segment and accelerationdirection one feature vector is extracted, containing one or more of the following codes bin LBP:

- The whole histogram: contains 256 values

- The uniform histogram: contain 59 out of 256 values

For the ECG signal, the features are extracted from each heartbeat ECG signal.

Here, $m$ corresponds to the data segment size. In this study, we used 8 for convenience to form uniform LBP from 256 bins.

Hence, the transformation codes are obtained for all the signal points, the objective is to obtain a feature representation that carries sufficiently high discriminating ability for reliable ECG or Gait based biometric

Additionally, we have doing many experiences to select the best combination of parameters of 1D-LBP

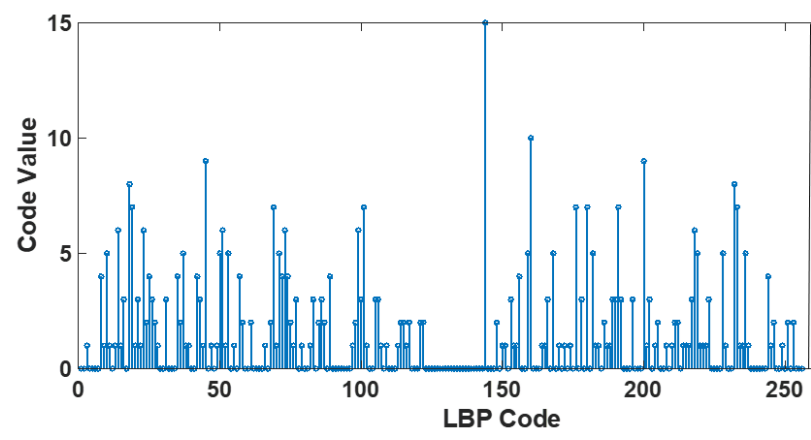

Figure.5 Calculating the LBP code by 1D-LBP in case the magnitude acceleration of Gait signal

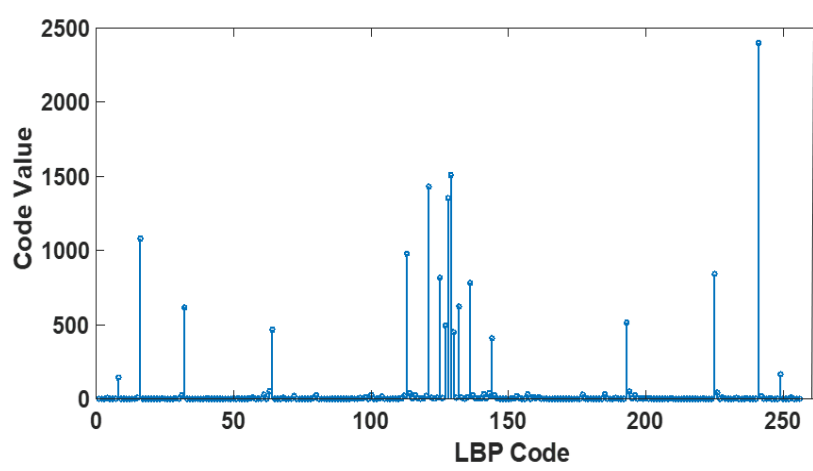

Figure.6 Calculating the LBP code by 1D-LBP in case the Heartbeat of ECG signal

such; segment length and overlapping percent for ECG and Gait signal. Fig. 5 and Fig. 6 show the results of 1D-LBP method, which was applied on the ECG and Gait signal.

\subsection{Features fusion}

A scores fusion system consists of two modules: a fusion module and a decision module. That is to say that, the identification task becomes a problem of classification, where two features are to be considered as client and the second as an impostor vectors for the same person. The fusion of features which compose the feature vectors is done in many different ways. In fact, there are serval methods as the mean, product, minimum, maximum or median, without requiring adaptation. However, there are also some advanced methods of combination require the setting of parameters such as the sum weighted. In this work, we have used a simple rule to merge both the two modalities as a single feature based the below formulation:

$$
V_{\text {ECG\&Gait }}=\left\{\text { Feature }_{\text {ECG }}, \text { Feature }_{\text {Gait }}\right\}
$$

\section{ECG and gait classification}

In this step, we have assessed the performance our proposed approach by using classifier KNN, 
PNN, SVM and RBF network [47]. The performances of the classifiers were evaluated in terms of accuracy and EER by Eq. 4 and Eq. 5 .

The KNN considered one the most well-known algorithm of classification/regression in the supervised of machine learning category which seek to produce an appropriate output (i.e., the class most common amongst its KNN measured by a distance function) when given new unlabelled data. In this study, the Euclidean distance method is used as the distance metric for KNN algorithm. The SVM classifier was initially designed to classify data instants into binary classes. The extension of SVM methods for multiclass classification problems has gained significant attention [37]. There are three main methods for implementing a multiclass SVM classifier, including Multiclass ranking SVMs, the pairwise coupling and one formulation for all. In our work, the best results are obtained when a Gaussian kernel is tested with One-against-all (OAA) SVMs for classification with a gamma value of 800 1000 and the Gaussian width value of $0.25 \sim 80$. For PNN and RBF classifiers has been successfully used in many applications such as pattern classification, data mining. RBF and PNN have a faster training procedure in comparison to multilayer perceptron trained using backpropagation (BP) algorithm. The structure of a PNN is similar to that of an RBF, both having a Gaussian spheroid activation function in the first of the two layers. While the output of the hidden layer is fed to the next layer, which is different for PNN (i.e., the parzen window) and RBF (generate linear decision boundaries, namely, hyperplanes). Training both PNN and RBF require the selection of parameters which influence the ensuing model performance. Therefore, to achieve a good model those parameters have to be chosen correctly. Examples, as stated earlier, are learning activation function [36], number of hidden (100 500) and the spread parameter $(\sigma)$ from 10 to 250 .

\section{Experimental results and discussion}

ECG-ID, ECG sinus [48] and Gait [49] public databases are used to validate and test our proposed work. On the ECG ID database, one ECG of each person is selected as the training samples set, with 90 signals in total. The last ECG of each person is selected as the testing samples set, with 90 signals in total. On the Sinus Rhythm Database, for each person, the ECG signal from hour 1 to hour 2 is used as training data, and the ECG signal from hour 13 to hour 14 is used as test data.

On the Gait database, the data are collected from 22 participants walking in the wild over a predefined path. The elements of this data set are composed of 4 attributes of real type, which are represented by: time-step, $\mathrm{x}, \mathrm{y}$ and $\mathrm{z}$ accelerations, for each time step. Time step values ranges from 0 to about $650 \mathrm{~s}$ depending on person walking. for each person, the magnitude of the gait signal from $10 \mathrm{~s}$ to hour $250 \mathrm{~s}$ is used as training data, and the ECG signal from 260s to $500 \mathrm{~s}$ is used as test data. In our multimodal system, an equal number of subjects must be performed.

The performance of a biometric system can be evaluated in terms of CRR (Correct Recognition Rate) and ERR (Equal Error Rate), which is given as:

$$
\begin{gathered}
C C R=\frac{\text { number of correctly identified subjects }}{\text { the total number of subjects }} \\
E E R=\frac{(F A R+F R R)}{2}
\end{gathered}
$$

Where: FRR indicates that genuine person was considered as imposter and FAR indicates that imposter was considered as genuine person. ERR indicates the point where: FRR-FAR $=0$. We also generate a ROC curve which to assess and comparing the performance our approach against conventional approach (unimodal system).

The classification accuracy achieved by 1D-LBP feature extraction techniques with different classifiers is shown in Fig 7 and Fig 8. Among the five machine learning classifiers used in this work in case ECG and Gait as unimodal system, it is found that $\mathrm{RBF}$ reached the highest classification accuracy.

From the Table 1, the obtained numerical results in term of CRR, ERR, FAR and FRR have shown that the proposed multimodal system is better than the unimodal system such as, ECG biometric system and Gait biometric system separately. (see Fig. 9)

As we mentioned in the previous sections, the effect of employing different values for parameters the segment length selection to have perform 1DLBP approach and the classification algorithms (PNN,

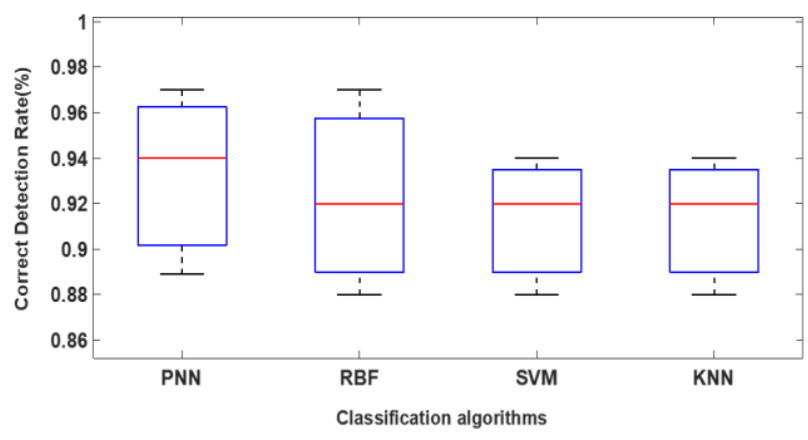

Figure.7 Comparison of the recognition performance for the 1DLBP technique on the ID database 


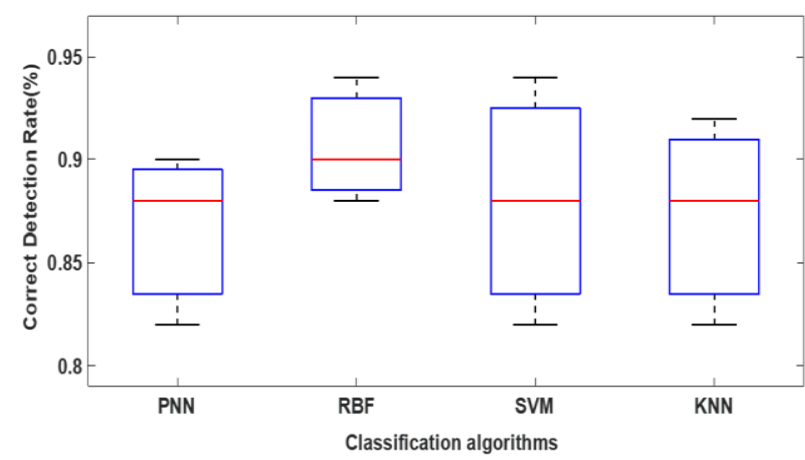

Figure.8 Comparison of the recognition performance for the 1DLBP technique on the gait database

Table 1. Performance evaluation of the proposed

\begin{tabular}{|c|c|c|c|}
\hline \multicolumn{4}{|c}{ approach } \\
\hline & Modality & EER & acc \\
\hline \multirow{4}{*}{ LBP } & ECG -ID & 3.95 & 94.44 \\
\cline { 2 - 4 } & ECG-sinus & 2.6 & $100 \%$ \\
\cline { 2 - 4 } & GAIT & 35.03 & 94.44 \\
\cline { 2 - 4 } & Fusion gait+ID & 3.90 & 94.44 \\
\cline { 2 - 4 } & Fusion gait+sinus & 2.60 & $100 \%$ \\
\hline
\end{tabular}

RBF, SVM, KNN) on the identification precision of the proposed system have been evaluated on the first fold.

The results use the equal error rate (EER), which refers to the intersection point between the false acceptance rate (FAR) and the false rejection rate (FRR) curves.

In the first experience, the results for the unimodal EEG and Gait approach are summarized. Higher results were obtained using the ECG signal component. From the unimodal ECG approach an $\mathrm{EER}=3.83 \%$ has been obtained. (see Fig. 10)

From the previous Figs. 7 and 8, it can be argued that the authentication result of the ECG based biometric with PNN is more robust than the unimodal system that used Gait for human identification. Also, we can prove that the proposed ECG based biometric can be used for authentication with acceptable authentication results.

In the second experience, we present the results of the score fusion of ECG and Gait for human authentication purpose. As can be seen in Table 1, we reported the results (in terms of EER) obtained from the fusion of ECG and ECG features. From the achieved results, it can be noticed that the ERR is reduced to $3.9 \%$ and $2.6 \%$ respectively, beside to $100 \%$ for accuracy.

Considering the obtained results, the proposed methods accuracy is comparable with all other methods is provided in Table 2 and as can be seen where our proposed bimodal method performs better than the other works. In this comparison, only the

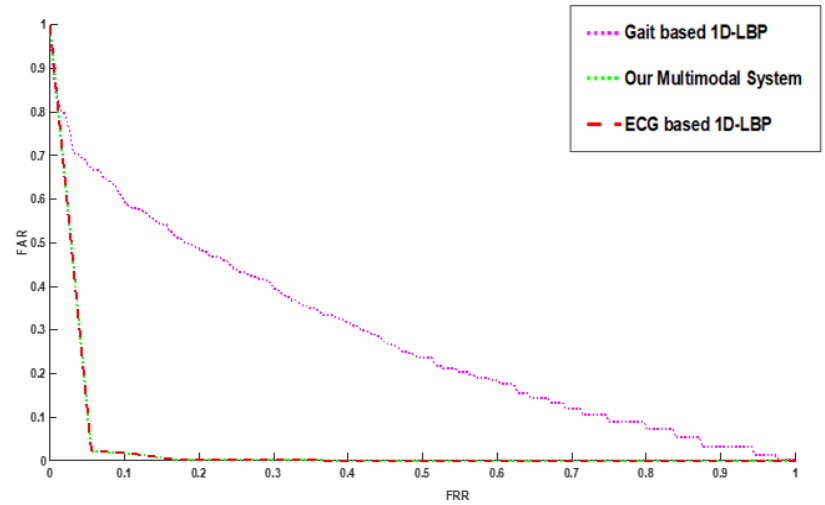

Figure.9 Roc curve for multimodal system and ECG(ID)Gait unimodal system separately

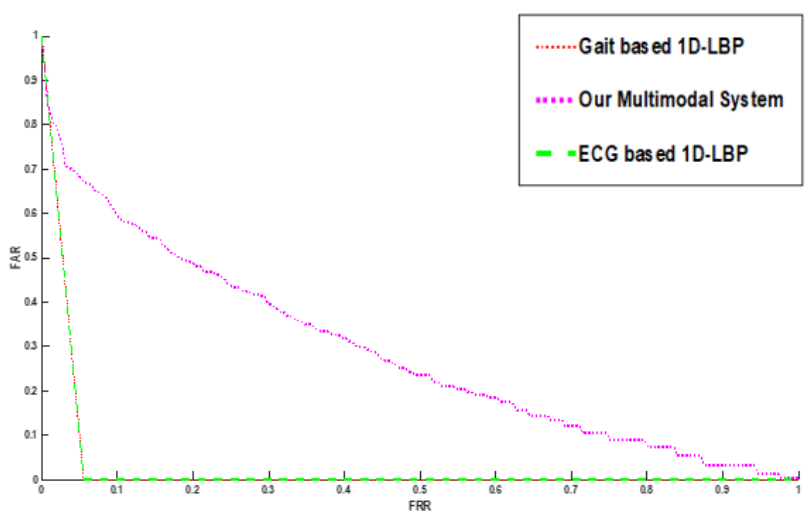

Figure.10 Roc curve for multimodal system and ECG(sinus)-Gait unimodal system separately

methods proposed in references [30] and our method used ECG and Gait, however, the most of the available methods have used the sufficient number of users, and this is why the most of multimodal frameworks more focused on the physical modalities such as face, iris, etc.

\section{Conclusion}

This paper proposes a new multibiometric system-based ECG and Gait for human authentication. Through our experiments, we concluded that behavioural biometric is an effective and powerful tool to be used as biometrics. This type of hidden biometric is relatively easy to acquire and simple to process due to its low dimensionality. The motivation behind this study is to improve conventional multibiometric methods which often use face and iris by investigating on the ECG and Gait signal. The both ECG and Gait signal present on each human activity either by his heart or manner of walk is extracted and used to generate a signature of each individual for biometrics purposes.

A novel multimodal system using 1D-LBP based on score level fusion of ECG and gait for human 
Table 2. Performance measures Comparison for our proposed multimodal systems against state-of-the-art multimodal systems

\begin{tabular}{|c|c|c|c|c|}
\hline \multicolumn{2}{|c|}{ References } & Modalities & $\begin{array}{l}\text { Fusin } \\
\text { level }\end{array}$ & $\begin{array}{c}\text { CRR } \\
\%\end{array}$ \\
\hline \multicolumn{2}{|c|}{ Israel et al. [26] } & $\begin{array}{l}\text { ECG and } \\
\text { face }\end{array}$ & decision & 94.6 \\
\hline \multicolumn{2}{|c|}{ Derawi et al. [30] } & $\begin{array}{l}\text { ECG and } \\
\text { Gait }\end{array}$ & features & ------- \\
\hline \multicolumn{2}{|c|}{ Belgacem et al. [50] } & $\begin{array}{c}\text { ECG and } \\
\text { EMG }\end{array}$ & features & 99.98 \\
\hline \multicolumn{2}{|c|}{$\begin{array}{c}\text { Boumbarov et al. } \\
\text { [51] }\end{array}$} & $\begin{array}{l}\text { ECG and } \\
\text { face }\end{array}$ & decision & 99.5 \\
\hline \multicolumn{2}{|c|}{$\begin{array}{l}\text { Al.hamdani et al } \\
{[52]}\end{array}$} & $\begin{array}{l}\text { ECG and } \\
\text { speech }\end{array}$ & score & ------- \\
\hline \multicolumn{2}{|c|}{ Barra et al [53] } & $\begin{array}{c}\text { ECG and } \\
\text { EEG }\end{array}$ & score & 96.79 \\
\hline \multicolumn{2}{|c|}{ Aghakabi et al. [54] } & $\begin{array}{c}\text { ECG, } \\
\text { Hand vein }\end{array}$ & decision & 94.7 \\
\hline \multicolumn{2}{|c|}{$\begin{array}{c}\text { Chakraborty et al } \\
{[55]}\end{array}$} & $\begin{array}{l}\text { ECG and } \\
\text { Face }\end{array}$ & feature & 97.5 \\
\hline \multirow{2}{*}{$\begin{array}{c}\text { Our } \\
\text { experime } \\
\text { nts }\end{array}$} & $1^{\text {st }}$ case & $\begin{array}{c}\text { ECG and } \\
\text { Gait }\end{array}$ & Feature & 100 \\
\hline & $2^{\text {nd }}$ case & $\begin{array}{l}\text { ECG and } \\
\text { Gait }\end{array}$ & Feature & 94.44 \\
\hline
\end{tabular}

authentication is presented in this work. The feature extraction for individual modalities are extracted using 1D-LBP and then biometric templates are generated from these features. After that, the machine learning as KNN, SVM, RBF and PNN are applied to perform the classification task.

The feature of ECG and Gait resulting from (1DLBP) are fused both to generate a unique template which presents the feature of subjects. The obtained results show that the proposed approach improves the accuracy of system to $100 \%$ and hence, it performs better than ECG and Gait biometric systems taken separately.

As a perspective, we propose to use this approach in an uncontrolled environment based on other biometric samples (EMG, EEG) in order to make the task of biometric technology more confidence and robust.

\section{References}

[1] A. K. Jain, P. Flynn, and A. A. Ross, Handbook of biometrics. Springer Science \& Business Media, 2007.

[2] P. Campisi, "Security and privacy in biometrics: towards a holistic approach", Security and Privacy in Biometrics, Springerp. 1-23, 2013.
[3] S. Dey and D. Samanta, Unimodal and Multimodal Biometric Data Indexing eBook Paket Gesamtpaket 2014.

[4] S. Eberz, K. B. Rasmussen, V. Lenders, and I. Martinovic, "Evaluating behavioral biometrics for continuous authentication: Challenges and metrics", In: Proc. of the 2017 ACM on Asia Conference on Computer and Communications Security, 2017, p. 386-399.

[5] I. Traore, M. Alshahrani, and M. S. Obaidat, "State of the art and perspectives on traditional and emerging biometrics: A survey", Secur. Priv., Vol. 1, No. 6, p. e44, 2018.

[6] B. P. Simon and C. Eswaran, "An ECG classifier designed using modified decision based neural networks", Comput. Biomed. Res., Vol. 30, No. 4, p. 257-272, 1997.

[7] A. A. Chlaihawi, B. B. Narakathu, S. Emamian, B. J. Bazuin, and M. Z. Atashbar, "Development of printed and flexible dry ECG electrodes", Sens. Bio-Sens. Res., Vol. 20, pp. 9-15, 2018.

[8] L. Biel, O. Pettersson, L. Philipson, and P. Wide, "ECG analysis: a new approach in human identification", IEEE Trans. Instrum. Meas., Vol. 50, No. 3, p. 808-812, 2001.

[9] T.-W. Shen, W. J. Tompkins, and Y.H. Hu, "One-lead ECG for identity verification", In: Proc. of Engineering in medicine and biology Proceedings of the Second Joint 24th Annual Conference and the Annual Fallmeeting of the Biomedical Engineering Society Embs/Bmes Conference, Vol. 1, p. 62-63, 2002.

[10] S. A. Israel, J. M. Irvine, A. Cheng, M. D. Wiederhold, and B. K. Wiederhold, "ECG to identify individuals", Pattern Recognit., Vol. 38, No. 1, p. 133-142, 2005.

[11] K. N. Plataniotis, D. Hatzinakos, and J. K. Lee, "ECG biometric recognition without fiducial detection", In: Proc. of Biometrics Symposium: Special Session on Research at the Biometric Consortium Conference, p. 1-6, 2006.

[12] G. Wübbeler, M. Stavridis, D. Kreiseler, R.-D. Bousseljot, and C. Elster, "Verification of humans using the electrocardiogram", Pattern Recognit. Lett., Vol. 28, No. 10, p. 1172-1175, 2007.

[13] D. Levine, J. Richards, and M. W. Whittle, Whittle's Gait Analysis-E-Book. Elsevier Health Sciences, 2012.

[14] D. Gafurov, "A survey of biometric gait recognition: Approaches, security and challenges", In: Proc. of Annual Norwegian Computer Science Conference, p. 19-21, 2007.

[15] P.-H. Chen, R.-L. Wang, D.-J. Liou and J.-S. Shaw, "Gait disorders in Parkinson's disease: 
assessment and management", Int. J. Gerontol., Vol. 7, No. 4, p. 189-193, 2013.

[16] P. Connor and A. Ross, "Biometric recognition by gait: A survey of modalities and features", Comput. Vis. Image Underst., Vol. 167, p. 1-27, 2018.

[17] C. BenAbdelkader, R. Cutler, H. Nanda, and L. Davis, "Eigen gait: Motion-based recognition of people using image self-similarity", In: Proc. of the International Conference on Audio-and Video-Based Biometric Person Authentication, p. 284-294, 2001.

[18] J. Wang, M. She, S. Nahavandi, and A. Kouzani, "A review of vision-based gait recognition mandhods for human identification", In: Proc. of the International Conference on Digital Image Computing: Techniques and Applications, p. 320-327, 2010.

[19] F. Tafazzoli and R. Safabakhsh, "Model-based human gait recognition using leg and arm movements", Eng. Appl. Artif. Intell., Vol. 23, No. 8, p. 1237-1246, 2010.

[20] M. Benouis, M. Senouci, R. Tlemsani and L. Mostefai, "Gait recognition based on modelbased methods and deep belief networks ", Int. J. Biom., Vol. 8, No. 3-4, p. 237-253, 2016.

[21] I. Rida, N. Almaadeed, and S. Almaadeed, "Robust gait recognition: a comprehensive survey", IET Biom., Vol. 8, No. 1, p. 14-28, 2018.

[22] H. J. Ailisto, M. Lindholm, J. Mantyjarvi, E. Vildjiounaite, and S.-M. Makela, "Identifying people from gait pattern with accelerometers", Biometric Technology for Human Identification II, Vol. 5779, p. 7-14, 2005.

[23] D. Gafurov, Performance and security analysis of gait-based user authentication, Doctoral Dissertation, University of Oslo, 2008.

[24] M. O. Derawi, C. Nickel, P. Bours, and C. Busch, "Unobtrusive user-authentication on mobile phones using biometric gait recognition", In: Proc. of 2010 Sixth International Conference on Intelligent Information Hiding and Multimedia Signal Processing, p. 306-311, 2010.

[25] I. Dehache and L. Souici-Meslati, "Immunological classifiers for accelerometerbased gait identification", Int. J. Biom., Vol. 9, No. 3, p. 225-242, 2017.

[26] S. A. Israel, W. T. Scruggs, W. J. Worek, and J. M. Irvine, "Fusing face and ECG for personal identification", In: Proc. of the 32nd Applied Imagery Pattern Recognition Workshop, p. 226231, 2003.

[27] V. Sireesha and S. R. K. Reddy, "Two Levels Fusion Based Multimodal Biometric
Authentication Using Iris and Fingerprint Modalities", International Journal of Intelligent Engineering and Systems, Vol. 9, No. 3, p. 2135, 2016.

[28] M. Akhavansaffar, A. Nakhaei, and M. M. Ardakan, "A Multimodal Biometric Authentication System Using Ear and Face", $J$. Comput., Vol. 13, No. 7, p. 876-889, 2018.

[29] T. K. Thivakaran, S. V. V. N. C. Padira, A. S. Kumar, and S. S. Reddy, "Fusion Based Multimodel Biometric Authentication System using Ear and Fingerprint", International Journal of Intelligent Engineering and Systems, Vol.12, No.1, pp. 62-73, 2019.

[30] M. Derawi and I. Voitenko, "Fusion of gait and ECG for biometric user authentication", In: Proc. of the International Conference of the Biometrics Special Interest Group (BIOSIG), p. 1-4, 2014.

[31] N. Belgacem, R. Fournier, A. Nait-Ali, and F. Bereksi-Reguig, "A novel biometric authentication approach using ECG and EMG signals", J. Med. Eng. Technol., Vol. 39, No. 4, p. 226-238, 2015.

[32] M. Regouid, M. Touahria, M. Benouis, and N. Costen, "Multimodal biometric system for ECG, ear and iris recognition based on local descriptors", Multimed. Tools Appl., p. 1-27, 2019.

[33] M. Hammad and K. Wang, "Parallel score fusion of ECG and fingerprint for human authentication based on convolution neural network", Comput. Secur., Vol. 81, p. 107-122, 2019.

[34] F. Miao, Y. Cheng, Y. He, Q. He, and Y. Li, “A wearable context-aware ECG monitoring system integrated with built-in kinematic sensors of the smartphone", Sensors, Vol. 15, No. 5, p. 11465 11484, 2015.

[35] R.W. Frischholz and U. Dieckmann, "BiolD: a multimodal biometric identification system IEEE Journals \& Magazine", Comput. J., Vol. 33, No. 2, p. 64-68, 2000.

[36] D. Kaur and G. Kaur, "Level of fusion in multimodal biometrics: A review", Int. J. Adv. Res. Comput. Sci. Softw. Eng., Vol. 3, No. 2, 2013.

[37] H. Sellahewa, N. Ibrahim, and S. Zeadally, "Biometric Authentication for Wearables", in Biometric-Based Physical and Cybersecurity Systems", Springer, p. 355-386, 2019.

[38] K. H. Chon and D. D. McManus, "Detection of atrial fibrillation using a smartwatch", Nat. Rev. Cardiol., Vol. 15, No. 11, p. 657, 2018. 
[39] M. Akay, Time Frequency and Wavelets in Biomedical Signal Processing. IEEE press series in Biomedical Engineering, 1998.

[40] M. Pietikäinen, A. Hadid, G. Zhao, and T. Ahonen, Computer vision using local binary patterns, Vol. 40. Springer Science \& Business Media, 2011.

[41] T. Ojala, M. Pietikäinen, and D. Harwood, "A comparative study of texture measures with classification based on featured distributions", Pattern Recognit., Vol. 29, No. 1, p. 51-59, 1996.

[42] M. Regouid and M. Benouis, "Shifted 1D-LBP Based ECG Recognition System", In: Proc. of the International Symposium on Modelling and Implementation of Complex Systems, p. 168-179, 2018.

[43] E.-S. El-Alfy and A. G. Binsaadoon, "Automated gait-based gender identification using fuzzy local binary patterns with tuned parameters", J. Ambient Intell. Humaniz. Comput., Vol. 10, No. 7, p. 2495-2504, 2019.

[44] Ö. F. Ertuğrul, Y. Kaya, R. Tekin, and M. N. Almal1, "Detection of Parkinson's disease by shifted one dimensional local binary patterns from gait", Expert Syst. Appl., Vol. 56, p. 156163, 2016.

[45] S. J. Orfanidis, Introduction to signal processing, Prentice-Hall, Inc., 1995.

[46] J. Pan and W. J. Tompkins, "A real-time QRS detection algorithm", IEEE Trans. Biomed. Eng., Vol. 32, No. 3, p. 230-236, 1985.

[47] S. Theodoridis, Machine learning: a Bayesian and optimization perspective. Academic Press, 2015.

[48] M. Merone, P. Soda, M. Sansone, and C. Sansone, "ECG databases for biometric systems: A systematic review", Expert Syst. Appl., Vol. 67, p. 189-202, 2017.

[49] P. Casale, O. Pujol, and P. Radeva, "Personalization and user verification in wearable systems using biometric walking patterns ", Pers. Ubiquitous Comput., Vol. 16, No. 5, p. 563-580, 2012.

[50] N. Belgacem, R. Fournier, A. Nait-Ali, and F. Bereksi-Reguig, "A novel biometric authentication approach using ECG and EMG signals", J. Med. Eng. Technol., Vol. 39, No. 4, p. 226-238, 2015.

[51] O. Boumbarov, Y. Velchev, K. Tonchev, I. Paliy, and G. Chetty, "Face and ECG based multimodal biometric authentication", Advanced Biometric Technologies, In Tech, 2011.

[52] O. Al-Hamdani, A. Chekima, J. Dargham, S. Salleh, F. Noman, H. Hussain, A. Ariff and A.
M. Noor, "Multimodal biometrics based on identification and verification system", J. Biom. Biostat., Vol. 4, No. 2, p. 1-8, 2013.

[53] S. Barra, A. Casanova, M. Fraschini, and M. Nappi, "Fusion of physiological measures for multimodal biometric systems", Multimed. Tools Appl., Vol. 76, No. 4, p. 4835-4847, 2017.

[54] A. Aghakabi and S. Zokaee, "Fusing dorsal hand vein and ECG for personal identification", In: Proc. of 2011 International Conference on Electrical and Control Engineering, p. 59335936, 2011.

[55] S. Chakraborty, M. Mitra, and S. Pal, "Biometric analysis using fused feature set from side face texture and electrocardiogram", IET Sci. Meas. Technol., Vol. 11, No. 2, p. 226-233, 2016. 